\title{
RESEARCH
}

Open Access

\section{Synthesising practice guidelines for the development of community-based exercise programmes after stroke}

\author{
Leon Poltawski ${ }^{*}$, Charles Abraham² ${ }^{2}$ Anne Forster ${ }^{3}$, Victoria A Goodwin ${ }^{1}$, Cherry Killbride ${ }^{4}$, Rod S Taylor ${ }^{1}$
} and Sarah Dean ${ }^{1}$

\begin{abstract}
Background: Multiple guidelines are often available to inform practice in complex interventions. Guidance implementation may be facilitated if it is tailored to particular clinical issues and contexts. It should also aim to specify all elements of interventions that may mediate and modify effectiveness, including both their content and delivery. We conducted a focused synthesis of recommendations from stroke practice guidelines to produce a structured and comprehensive account to facilitate the development of community-based exercise programmes after stroke.
\end{abstract}

Methods: Published stroke clinical practice guidelines were searched for recommendations relevant to the content and delivery of community-based exercise interventions after stroke. These were synthesised using a framework based on target intervention outcomes, personal and programme proximal objectives, and recommended strategies.

Results: Nineteen guidelines were included in the synthesis (STRIDES; STroke Rehabilitation InterventionDevelopment Evidence Synthesis). Eight target outcomes, 14 proximal objectives, and 94 recommended strategies were identified. The synthesis was structured to present best practice recommendations in a format that could be used by intervention programme developers. It addresses both programme content and context, including personal factors, service standards and delivery issues. Some recommendations relating to content, and many relating to delivery and other contextual issues, were based on low level evidence or expert opinion. Where opinion varied, the synthesis indicates the range of best practice options suggested in guidelines.

Conclusions: The synthesis may assist implementation of best practice by providing a structured intervention description that focuses on a particular clinical application, addresses practical issues involved in programme development and provision, and illustrates the range of best-practice options available to users where robust evidence is lacking. The synthesis approach could be applied to other areas of stroke rehabilitation or to other complex interventions.

Keywords: Guidelines, Synthesis, Stroke, Rehabilitation, Exercise

\footnotetext{
* Correspondence: L.Poltawski@exeter.ac.uk

'University of Exeter Medical School, Veysey Building, Salmon Pool Lane, Exeter EX2 4SG, UK

Full list of author information is available at the end of the article
} 


\section{Background}

The evidence base for rehabilitation after stroke is expanding rapidly [1]. Drawing on this evidence, numerous guidelines have been developed by expert bodies, to facilitate development of best clinical practice $[2,3]$. Many guidelines are comprehensive in coverage, addressing all phases in recovery from stroke and the many disciplines involved in providing post-stroke care [2-4]. However, the existence of practice guidelines does not in itself guarantee their implementation. Even high quality guidelines, developed according to rigorous standards and presenting unambiguous recommendations based upon robust evidence, may fail to influence practice for a variety of reasons. These may include factors intrinsic to the guidelines themselves, such as their user-friendliness and relevance to the user organisation [5-7]. They may also relate to the environment in which they are applied, for example organisational structures, service resource limitations and professional awareness [6,8-10].

A number of strategies have been suggested to reduce barriers to guideline implementation. One is to provide guidelines in multiple formats targeting different professional groups; some stroke practice guidelines do this by providing separate listings of recommendations for nursing and allied health professionals [4]. Another strategy is to adapt guidelines according to context such as a particular healthcare setting or application $[9,10]$. This involves identifying a clinical application or question, extracting relevant recommendations from existing guidelines, and re-presenting them in a format that is appropriate to the context [11]. Adapting existing guidelines enables consideration of a variety of recommendations produced in different cultural and organisational contexts, and avoids unnecessary duplication of effort [11]. The ADAPTE initiative has developed formal and detailed methods for this process [12]. However, it provides limited guidance on the process of 'customisation' - integrating recommendations from guidelines with varying concerns and terminologies and which may not explicitly address the application of interest. This is particularly pertinent when guidelines are being adapted to inform the development of complex interventions that involve multiple components and delivery issues. In such cases, adapting existing guidelines involves the synthesis of recommendations into an overarching structure selected to facilitate programme development. We use the term 'synthesis' because the process goes beyond the extraction and reordering of practice recommendations; it requires the development of a thematic framework into which the recommendations are placed, and may involve an element of conceptual translation.

The synthesis of practice guidelines for specific clinical applications is relatively uncommon. A recent example focused on the assessment and management of low back pain [13]. Using a systematic review methodology, data were extracted from ten guidelines and synthesised to produce tables of recommended diagnostic and treatment options, arranged under headings of primary and secondary care. None of the individual guidelines contained all of these recommendations, which supports the case for synthesising multiple publications. The authors note the lack of guidance on the quantity or 'dose' of therapy, and suggest that guidelines often ignore practical realities faced by clinicians [13]. The synthesis did not address issues such as the clinical setting, who provides the interventions, and what level of expertise is required [9]. In rehabilitation, these and other contextual factors such as personal beliefs, differences in goals, and power relationships between therapists and client can significantly influence outcomes [14-16]. Theoretical models have been developed indentifying contextual factors as both moderators and mediators of rehabilitation outcomes $[17,18]$, and guidelines for the development and evaluation of complex interventions suggest that these factors should be identified and described in accounts of interventions $[19,20]$. These principles are also supported by realist approaches to evaluation and evidence synthesis for complex interventions, in which effectiveness is seen as highly contingent on context: how, to whom, and in what circumstances interventions are implemented [21,22].

Thus, there is a need for application-specific guidelines that address both the content of interventions and the context in which they are delivered. A synthesis of guidelines created for this purpose [7,16-22] may be more credible and useful to potential users since it is tailored to a particular need and takes account of the many factors that may influence outcome [12]. Synthesising guidelines for the purpose of programme development is not a well-developed methodology, and there is a need to gain and reflect on experiences of doing so. We conducted a synthesis as part of development work for a clinical trial of a community-based exercise programme for stroke survivors. Such programmes are increasingly being offered to facilitate regular engagement in exercise by long term stroke survivors and so to improve their health outcomes [23-26], but the evidence for particular types of multi-component programme is presently limited. To help develop an intervention that is congruent with current best evidence, we synthesised relevant guideline recommendations regarding programme content, delivery and other contextual factors. The aim was to develop a synthesis that places recommendations in a conceptually coherent structure that can inform programme development. The purpose of this paper is to describe the process and outcome of the synthesis, and to discuss the potential value of this approach to others who wish to develop comprehensive syntheses of 
guidelines to assist the development and evaluation of other forms of complex intervention.

\section{Methods}

The synthesis used some of the elements described in the ADAPTE approach: defining the health question, searching for and screening guidelines, selecting recommendations, and creating a customised guideline [12] (see Figure 1). Data extraction and the synthesis process were informed by an approach called Intervention Mapping [27], which has been used by others to structure the development of complex health interventions [27-31], and is described later in this section.

\section{Defining the health question}

The question we addressed in this synthesis was: how should community-based exercise programmes for long term stroke survivors be structured, in terms of content and delivery, to maximise their effectiveness?

\section{Search for guidelines}

We searched for stroke practice guidelines (sets of recommendations generated by expert panels, regarding the content and delivery of interventions) published between 2000 and July 2012. We consulted the Medline and Cinahl bibliographic databases, the National Guideline Clearinghouse (www.guideline.gov), and Google Scholar. The search strategy used combinations and variations of the terms 'practice guideline,' 'stroke,' 'rehabilitation' and 'exercise', and is described in Additional file 1. Free text searches using Google were also employed.

\section{Eligibility criteria}

Guidelines were included if they made recommendations regarding either the use of physical exercise by adults after stroke, or the delivery of such interventions through community-based programmes. Exercise was defined as the use of structured, repetitive physical activities to maintain or enhance physical functioning [32]. Only guidelines developed or endorsed by national or professional organisations or bodies were included, and those concerned solely with acute care or primary prevention were excluded. Due to resource limitations, we excluded publications that were not available in English or which had to be purchased. Where guidelines had been superseded by, or incorporated into, a more recent publication from the same organisation, the latest publication was used. One reviewer (LP) conducted the search, and two (LP and SD) discussed and agreed which guidelines should be included in the synthesis. The ADAPTE process advocates quality assessment of guidelines, and suggests that quality scores may be used as eligibility criteria [12]. However, we decided that, for the purposes of this synthesis, the strength of evidence for recommendations was the key factor in informing practice, and so focussed on this rather than the methodological quality of the guidelines themselves.

\section{Data extraction}

Both data extraction and analysis drew on an approach developed to assist in the planning of complex health interventions, called Intervention Mapping [27]. This approach proposes that interventions are characterised in

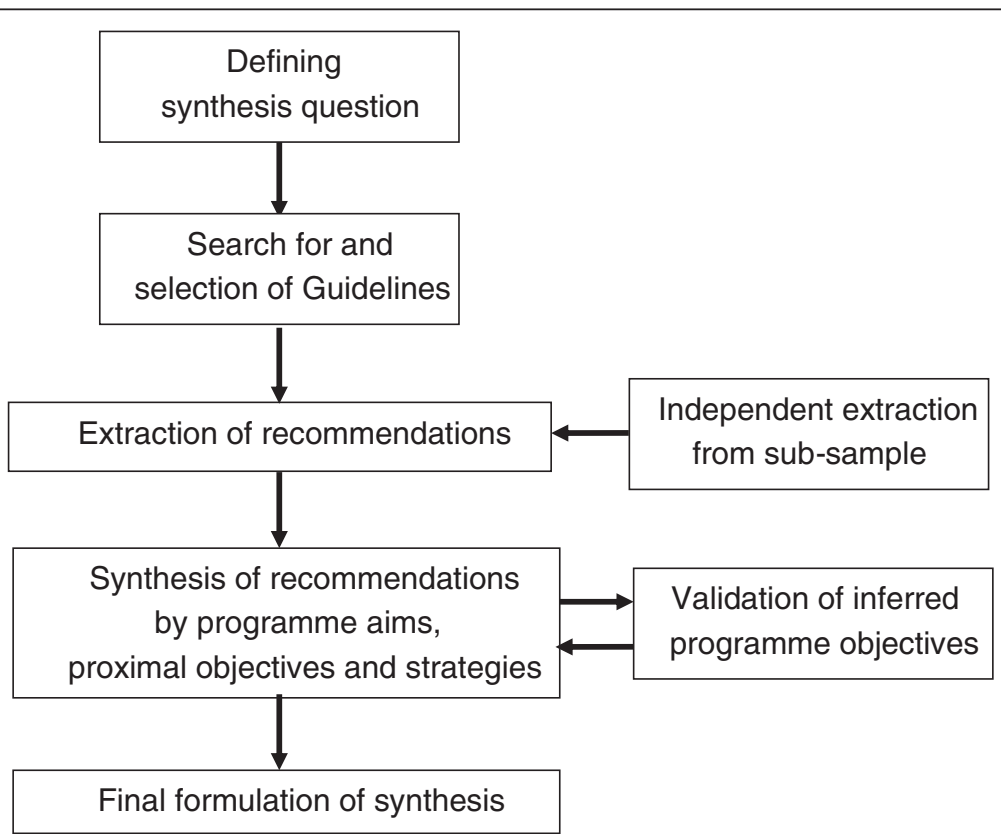

Figure 1 Synthesis process. 
terms of target outcomes, proximal objectives, methods and practical strategies. Target outcomes are the long term benefits that the intervention is designed to achieve for its participants; proximal objectives are the changes in the individual participant and their environment that are necessary to obtain the outcomes; and practical strategies are the means (or delivery modes) that may be employed to serve these objectives [27]. In Intervention Mapping, methods and strategies follow from a theoretical understanding of underlying regulatory processes. The approach has been used to aid the development of a range of complex health interventions, and it specifically seeks to incorporate service delivery and other contextual factors into intervention descriptions [30,31]. We used its principles to guide data extraction by selecting statements or recommendations relating to the aims, objectives, content and delivery of exercise-based interventions. Although the approach also involves the identification of theoretical underpinnings for strategies [27], we did not extract statements relating to theory in guidelines because we were primarily concerned with practice recommendations.

Our focus was on exercise programmes that can be delivered in community settings without the requirement for specialist clinical equipment. Therefore, we did not extract recommendations relating to the use of robots, partial-bodyweight support therapy, mirror boxes etc., but did include those requiring equipment that would be available in a typical community-based gym. Where guidelines cited and/or graded evidence in support of their recommendations, the type and grading of the evidence was recorded. We included both research-evidenced recommendations and those based on expert consensus alone because, in the absence of robust evidence, programme planners and practitioners may still be guided by the experience and views of experts. One reviewer (LP) extracted data from all guidelines, and a second reviewer (SD) verified the process by independent data extraction from four of the guidelines. Any differences were discussed by the reviewers, and additional material was included if agreed to be relevant.

\section{Synthesis of recommendations}

Data were analysed thematically, using a form of template analysis in which data are initially. Categorised using predefined themes and then organised iteratively under a hierarchy of emerging sub-themes [33]. In this case, the predefined themes used Intervention Mapping terms: target outcomes, proximal objectives and strategies. Most of the guidelines were not specifically concerned with community-based exercise programmes, and few made explicit statements regarding the proximal objectives of such programmes. Thus, most data were initially classified under the 'strategies' theme. Using an iterative approach, these strategies were grouped and regrouped into a series of sub-themes, which were then expressed as proximal objectives.

One reviewer (LP) conducted the first iteration of this process using all the extracted data; subsequent iterations were developed through discussion with three other reviewers (SD, VG, CK) with rehabilitation expertise and who were familiar with a sample of the included guidelines, and one reviewer (CA) with expertise in Intervention Mapping methodology. To validate the inference of proximal objectives from the recomendations, two reviewers (LP and SD) independently inspected four guidelines to judge whether the proximal objectives were explicitly or implicitly present in them.

The guidelines used a variety of systems to grade the evidence cited to support their recommendations. For the synthesis, the gradings were reclassified under a single simple system that had been employed by one of the guidelines [34]: multiple randomised control trials (RCTs) and meta-analyses are given the highest grading (1), followed by single RCTs and non-randomised studies (2), and finally expert consensus, case studies, and standards of care (3).

\section{Results}

The search of bibliographic databases identified 135 potentially relevant publications, which were screened initially by title and abstract, then by full text (see Figure 2). A total of 13 guidelines meeting the eligibility criteria were included, along with 6 further guidelines obtained through Google free text searches [1-3,34-49]. They were from Australia, Canada, a European network, the Netherlands, New Zealand, Singapore, South Africa, the United Kingdom and the United States. Three of these were produced by the American Heart Association but targeted different audiences with distinct recommendations. The guidelines varied considerably in scope, some providing only general endorsement of physical exercise (e.g., [36,44]), others making more specific and detailed recommendations $[2,3]$. The median number of relevant recommendations abstracted per guideline was 15 , although 2 guidelines made only one recommendation each relevant to the synthesis topic $[46,49]$.

The guidelines used a variety of formats, e.g., being structured according to the phases of stroke recovery, the concerns of each health professional group, or the types of intervention that could be provided. Terminologies also differed, for example in the naming of forms of exercise or types of health practitioner. Most addressed the continuum of care from acute to chronic phases, but two focused specifically on community-based interventions for longer-term stroke survivors [35,42]. These provided the majority of delivery-related recommendations, but did 


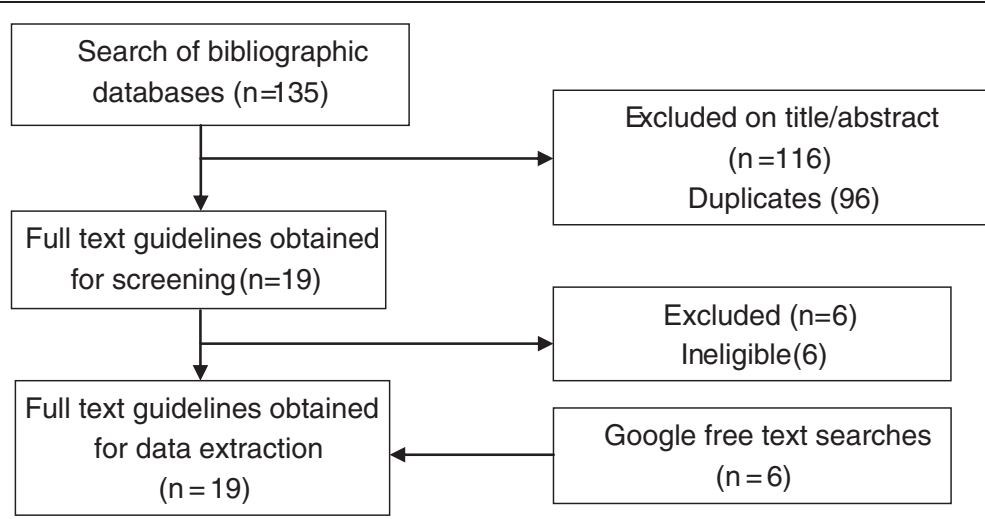

Figure 2 Search and screening flow chart.

not mention several content and delivery strategies suggested in other guidelines, such as the use of mental rehearsal and tele-training. Eight target outcomes of exercise-based interventions were explicitly identified in one or more guidelines (see Table 1). Three of these related to body structure and function, and five to activities and participation in life roles. A total of 94 recommendations were identified, and 14 proximal objectives were either explicitly stated in at least one guideline or formulated from themes emerging from the data synthesis.

Tables 2 and 3 summarise the proximal objectives and strategies for programme content and delivery respectively. Recommendations relating to contextual factors appear in both tables: environmental factors (such as family support) are addressed in Table 2, while personal factors (such as individual attitudes) are addressed by several strategies linked to objectives 6,7 and 8 . The tables also indicate the highest level of evidence cited in any guideline for each strategy. The strength of supporting evidence varied considerably: many of the recommendations for content were based on systematic reviews, whereas most of those relating to service delivery relied on qualitative studies or expert opinion. More detailed tables, providing page and section references for each recommendation, and any evidence gradings provided in each guideline, are available in Additional files 2 and 3 respectively.

Table 1 Target programme outcomes

\begin{tabular}{ll}
\hline Reduced risk of stroke & Optimisation of functional ability \\
{$[2,34,36,37,39,40,45,47,48]$} & {$[2,3,34,37,40,45]$} \\
Increased cardiovascular & Social participation to the extent desired \\
fitness $[2,35,37,39,40,44]$ & {$[45,48]$} \\
Enhanced mobility $[2,36,37]$ & $\begin{array}{l}\text { Life-long involvement in regular physical } \\
\text { exercise }[2,35,41,42,45,47,48]\end{array}$ \\
Reduced risk of falls & Self-management of physical exercise \\
{$[34,36,37,44]$} & {$[48]$} \\
\hline
\end{tabular}

\section{Programme content}

Seven proximal objectives focusing on the individual participant, along with 38 associated strategies, were identified in the synthesis. The objectives related to both physical outcomes, such as strength, aerobic fitness and functional ability, and to psychological outcomes, such as motivation and exercise self-management skills. The most commonly recommended exercises - with the highest level evidence - focussed on upper and lower limb strengthening, cardiovascular fitness and taskrelated practice. Treadmill training was frequently recommended both for improving aerobic endurance and to enhance walking performance. Several guidelines recommended and cited high level evidence for balance training, but there was less agreement about other sensorimotor strategies, e.g., mental rehearsal of movements and tasks, which received strong support from some guidelines $[43,45]$ but less from others $[2,42]$. Stretching and range of movement exercises were advocated by several, but had the lowest levels of supporting evidence.

Several guidelines recommended a combination of aerobic endurance, strength, functional practice, and balance exercises for long term stroke survivors $[3,37,40,43]$, but one claimed that there was no strong evidence to recommend any particular intervention in the long term ( $>1$ year) phase [44]. Numerous behaviour change techniques and strategies, such as motivational interviewing and active problem-solving, were recommended, but these generally relied on weaker or less stroke-specific evidence.

\section{Programme delivery}

Eight proximal objectives concerned with programme delivery were identified in the synthesis. These ranged from ensuring that activities are delivered in sufficient quantity to achieve a training effect, to providing programme governance. In total, 57 associated strategies were found. The most commonly recommended and highly evidenced were involving family and carers in the programme, and adapting content and delivery to the 
Table 2 Proximal objectives and strategies for programme content

\begin{tabular}{|c|c|c|c|c|c|c|c|c|c|c|c|c|c|c|c|c|c|c|c|c|c|c|}
\hline \multirow{2}{*}{\multicolumn{2}{|c|}{$\begin{array}{l}\text { Proximal objectives \& } \\
\text { recommended strategies }\end{array}$}} & \multirow{2}{*}{ Ev } & \multirow{2}{*}{ count } & \multirow{2}{*}{$\begin{array}{c}\mathrm{Au} \\
{[41]}\end{array}$} & \multirow{2}{*}{$\begin{array}{l}\text { Ca1 } \\
{[37]}\end{array}$} & \multirow{2}{*}{$\begin{array}{l}\mathrm{Ca} 2 \\
{[42]}\end{array}$} & \multirow{2}{*}{$\begin{array}{l}\text { Ca3 } \\
{[38]}\end{array}$} & \multirow{2}{*}{$\begin{array}{l}\text { Ca4 } \\
{[43]}\end{array}$} & \multirow{2}{*}{$\begin{array}{l}\text { Ca5 } \\
{[1]}\end{array}$} & \multirow{2}{*}{$\begin{array}{c}\mathrm{Eu} \\
{[44]}\end{array}$} & \multirow{2}{*}{$\begin{array}{l}\mathrm{Ne} \\
{[3]}\end{array}$} & \multirow{2}{*}{$\begin{array}{c}\mathrm{NZ} \\
{[48]}\end{array}$} & \multirow{2}{*}{$\begin{array}{c}\text { SA } \\
{[36]}\end{array}$} & \multirow{2}{*}{$\begin{array}{c}\mathrm{Si} \\
{[49]}\end{array}$} & \multirow{2}{*}{$\begin{array}{l}\text { UK1 } \\
{[45]}\end{array}$} & \multirow{2}{*}{$\begin{array}{l}\text { UK2 } \\
{[47]}\end{array}$} & \multirow{2}{*}{$\begin{array}{l}\text { UK3 } \\
{[35]}\end{array}$} & \multirow{2}{*}{$\begin{array}{l}\text { US1 } \\
\text { [39] }\end{array}$} & \multirow{2}{*}{$\begin{array}{l}\text { US2 } \\
{[40]}\end{array}$} & \multirow{2}{*}{$\begin{array}{l}\text { US3 } \\
{[34]}\end{array}$} & \multirow{2}{*}{$\begin{array}{c}\text { US4 } \\
{[2]}\end{array}$} & \multirow{2}{*}{$\begin{array}{l}\text { US5 } \\
{[46]}\end{array}$} \\
\hline & & & & & & & & & & & & & & & & & & & & & & \\
\hline 1 & Increase muscle strength & & 10 & $x$ & & $x$ & & $x$ & & & $x$ & $x$ & & & $x$ & & $x$ & $x$ & $x$ & & $x$ & \\
\hline & $\begin{array}{l}\text { a. Lower limb } \\
\text { strengthening }\end{array}$ & 1 & 8 & & & $x$ & & $x$ & $x$ & & $x$ & & & & & & $x$ & & $x$ & $x$ & $x$ & \\
\hline & $\begin{array}{l}\text { b. Upper limb } \\
\text { strengthening }\end{array}$ & 1 & 10 & & $x$ & $x$ & & $x$ & $x$ & & $x$ & & & & $x$ & & $x$ & & $x$ & $x$ & $x$ & \\
\hline & $\begin{array}{l}\text { c. Trunk and core muscle } \\
\text { strengthening }\end{array}$ & 2 & 3 & & & $x$ & & & & & & & & & & & $x$ & & $x$ & & & \\
\hline 2 & $\begin{array}{l}\text { Increase aerobic } \\
\text { endurance }\end{array}$ & & 12 & & $x$ & $x$ & & $x$ & $x$ & $x$ & $x$ & $x$ & & & $x$ & & $x$ & $x$ & $x$ & & $x$ & \\
\hline & a. Treadmill training & 1 & 6 & & $x$ & $x$ & & $x$ & & & $x$ & & & & & & & & $x$ & & $x$ & \\
\hline & b. Use of static bicycle & 1 & 3 & & & $x$ & & & & & & & & & & & $x$ & & $x$ & & & \\
\hline & $\begin{array}{l}\text { c. Other large muscle } \\
\text { group aerobic activity }\end{array}$ & 2 & 2 & & & $x$ & & & & & & & & & & & $x$ & & & & & \\
\hline 3 & $\begin{array}{l}\text { Regain and maintain } \\
\text { normal joint range of } \\
\text { movement }\end{array}$ & & 5 & & & $x$ & & & & & & & & & $x$ & & $x$ & & $x$ & & $x$ & \\
\hline & a. Lower limb stretching & 2 & 3 & & $x$ & $x$ & & & & & & & & & & & $x$ & & & & & \\
\hline & b. Upper limb stretching & 1 & 4 & & $x$ & $x$ & & & $x$ & & $x$ & & & & & & & & & & & \\
\hline & c. Trunk stretching & 2 & 2 & & & $x$ & & & & & & & & & & & $x$ & & & & & \\
\hline & $\begin{array}{l}\text { d. Lower limb movement } \\
\text { through range }\end{array}$ & 2 & 2 & & $x$ & & & & & & & & & & & & $x$ & & & & & \\
\hline & $\begin{array}{l}\text { e. Upper limb movement } \\
\text { through range }\end{array}$ & 2 & 3 & & $x$ & & $x$ & & & & & & & & & & $x$ & & & & & \\
\hline & $\begin{array}{l}\text { f. Unspecified stretch/ } \\
\text { range of movement } \\
\text { exercises }\end{array}$ & 3 & 5 & & & $x$ & $x$ & & & & & & & & $x$ & & & & $x$ & & $x$ & \\
\hline 4 & $\begin{array}{l}\text { Enhance sensorimotor } \\
\text { functions required for } \\
\text { functional activity }\end{array}$ & & 7 & & $x$ & $x$ & $x$ & & & & & & & & $x$ & & $x$ & $x$ & $x$ & & & \\
\hline & $\begin{array}{l}\text { a. Standing balance } \\
\text { training }\end{array}$ & 1 & 5 & & & $x$ & & $x$ & $x$ & & & & & & & & $x$ & & & & $x$ & \\
\hline & b. Sitting balance training & 1 & 4 & & & $x$ & & $x$ & & & $x$ & & & & & & & & & & $x$ & \\
\hline & c. Aquatic balance training & 1 & 2 & & & & & & $x$ & & & & & & & & & & & & $x$ & \\
\hline & $\begin{array}{l}\text { d. Unspecified balance } \\
\text { training }\end{array}$ & 1 & 3 & & & & & & $x$ & & & & & & $x$ & & & & $x$ & & & \\
\hline & $\begin{array}{l}\text { e. Cyclic movements of } \\
\text { paretic arm }\end{array}$ & 2 & 3 & & & & & & & & $x$ & & & & & & $x$ & & & & $x$ & \\
\hline & $\begin{array}{l}\text { f. Proprioceptive \& } \\
\text { kinaesthetic training }\end{array}$ & 2 & 6 & & $x$ & & $x$ & $x$ & & & $x$ & $x$ & & & & & & & & & $x$ & \\
\hline & $\begin{array}{l}\text { g. Mental rehearsal of } \\
\text { upper limb movements }\end{array}$ & 1 & 8 & $x$ & $x$ & & $x$ & $x$ & $x$ & & & $x$ & & & $x$ & & & & & & $x$ & \\
\hline 5 & $\begin{array}{l}\text { Enhance functional } \\
\text { ability }\end{array}$ & & 12 & $x$ & $x$ & $x$ & $x$ & $x$ & & & $x$ & $x$ & & & $x$ & & $x$ & & $x$ & $x$ & $x$ & \\
\hline & $\begin{array}{l}\text { a. Standing up \& sitting } \\
\text { down practice }\end{array}$ & 1 & 7 & & $x$ & $x$ & & $x$ & & & $x$ & $x$ & & & $x$ & & $x$ & & & & & \\
\hline & $\begin{array}{l}\text { b. Step training / stair } \\
\text { climbing }\end{array}$ & 1 & 5 & & & $x$ & & $x$ & & & & & & & & & $x$ & & $x$ & & $x$ & \\
\hline & $\begin{array}{l}\text { c. Walking (including } \\
\text { treadmill training) }\end{array}$ & 1 & 11 & $x$ & $x$ & $x$ & & $x$ & $x$ & & & $x$ & & & $x$ & & $x$ & & $x$ & $x$ & $x$ & \\
\hline & $\begin{array}{l}\text { d. Walking with rhythmic } \\
\text { cueing }\end{array}$ & 1 & 6 & $x$ & & & & $x$ & $x$ & & $x$ & $x$ & & & & & & & & & $x$ & \\
\hline & $\begin{array}{l}\text { e. Paretic upper limb task- } \\
\text { related training }\end{array}$ & 1 & 11 & $x$ & $x$ & & $x$ & $x$ & $x$ & & $x$ & $x$ & & & $x$ & & $x$ & & & $x$ & $x$ & \\
\hline
\end{tabular}


Table 2 Proximal objectives and strategies for programme content (Continued)

\begin{tabular}{|c|c|c|c|c|c|c|c|c|c|c|c|c|}
\hline & $\begin{array}{l}\text { f. Bilateral upper limb } \\
\text { task-related training }\end{array}$ & 1 & 5 & $x$ & & $x$ & $x$ & $x$ & & & & $x$ \\
\hline \multirow[t]{8}{*}{6} & $\begin{array}{l}\text { Establish \& maintain } \\
\text { motivation for regular } \\
\text { physical exercise }\end{array}$ & & 6 & $x$ & $x$ & & $x$ & $x$ & $x$ & $x$ & & \\
\hline & $\begin{array}{l}\text { a. Address personal beliefs } \\
\text { \& attitudes }\end{array}$ & 2 & 1 & & & & & & & $x$ & & \\
\hline & $\begin{array}{l}\text { b. Promote personal goal- } \\
\text { setting }\end{array}$ & 2 & 3 & & & & $x$ & $x$ & & $x$ & & \\
\hline & $\begin{array}{l}\text { c. Use motivational } \\
\text { interviewing }\end{array}$ & 2 & 1 & & & & & $x$ & & & & \\
\hline & $\begin{array}{l}\text { d. Promote use of } \\
\text { personal reflective diaries }\end{array}$ & 3 & 1 & & & & & & & $x$ & & \\
\hline & $\begin{array}{l}\text { e. Promote use of personal } \\
\text { exercise record including } \\
\text { repetitions, load and time } \\
\text { spent }\end{array}$ & 3 & 2 & & & & & & & $x$ & $x$ & \\
\hline & f. Use positive feedback & 2 & 1 & & & & & $x$ & & & & \\
\hline & g. Emphasise enjoyment & 3 & 2 & & $x$ & & & & & $x$ & & \\
\hline \multirow[t]{7}{*}{7} & $\begin{array}{l}\text { Develop self- } \\
\text { management skills for } \\
\text { ongoing physical exercise }\end{array}$ & & 1 & & $x$ & & & & & & & \\
\hline & $\begin{array}{l}\text { a. Educate for self- } \\
\text { monitoring for adverse } \\
\text { events }\end{array}$ & 3 & 1 & & & & & & & $x$ & & \\
\hline & $\begin{array}{l}\text { b. Promote active } \\
\text { problem-solving }\end{array}$ & 3 & 3 & & & & $x$ & $x$ & & & & $x$ \\
\hline & $\begin{array}{l}\text { c. Develop self-efficacy } \\
\text { skills }\end{array}$ & 3 & 2 & & $x$ & & & $x$ & & & & \\
\hline & $\begin{array}{l}\text { d. Encourage self- } \\
\text { monitoring to set } \\
\text { appropriate exercise levels }\end{array}$ & 3 & 2 & & $x$ & & & & & $x$ & & \\
\hline & $\begin{array}{l}\text { e. Encourage independent } \\
\text { practice of exercises }\end{array}$ & 3 & 2 & & $x$ & & & & & $x$ & & \\
\hline & $\begin{array}{l}\text { f. Provide guidance } \\
\text { booklets }\end{array}$ & 3 & 1 & & & & & & & $x$ & & \\
\hline
\end{tabular}

Ev $=$ highest level of evidence presented for strategy.

$\mathrm{n}=$ number of guidelines explicitly including objective or strategy.

$\mathrm{Au}=$ Australia; $\mathrm{Ca}=$ Canada; $\mathrm{Eu}=$ Europe; $\mathrm{Ne}=$ Netherlands, $\mathrm{NZ}=$ New Zealand; $\mathrm{SA}=$ South Africa; $\mathrm{Si}=$ Singapore

individual's circumstances and goals. However, there was inconsistency between guidelines on a number of recommendations. For instance, group classes conducted in fitness clubs or community centres were recommended by some [35,42], whereas others suggested that individual or home-based training may be preferable, and cited higher level research evidence in support of their recommendation $[3,39,48]$. Dosage recommendations also differed between guidelines, both in terms of frequency and intensity of exercises. Descriptors for appropriate intensity levels, for example, varied between 'slightly breathless' [45] and 'aggressive' [40], the latter level being specifically recommended for longer term stroke survivors [40].

\section{Synthesis methods}

In total, 6 of the 19 included guidelines were found via free text Google searches rather than bibliographic databases. These included the two guidelines providing most of the recommendations on programme delivery $[35,42]$. Intervention aims were specified in several guidelines and required only minor reformulation to be expressed as target objectives. Objectives were explicitly stated in few guidelines, and most of these referred to the content of programmes rather than their delivery. Therefore, it was necessary to impute several deliveryrelated objectives. Levels of agreement between the two researchers on the presence or absence of these objectives in four of the guidelines were good, with agreement on all objectives for two of the guidelines [35,40], on 13 out of 14 in one [2] and on 12 out of 14 in another [42].

Explicit links between aims and objectives were rarely made in the guidelines, and none were made in the synthesis. However, strategies were linked to particular content-related objectives in many guidelines, and these 
Table 3 Proximal objectives and strategies for programme delivery

\begin{tabular}{llllllllllllllllllllll}
\hline $\begin{array}{l}\text { Proximal objectives \& } \\
\text { recommended strategies }\end{array}$ & Ev & $\mathbf{n}$ & $\begin{array}{c}\text { Au } \\
\text { rea1 }\end{array}$ & Ca2 & Ca3 & Ca4 & Ca5 & Eu & Ne & NZ & SA & Si & UK1 & UK2 & UK3 & US1 & US2 & US3 & US4 & US5 \\
& & & {$[41]$} & {$[37]$} & {$[42]$} & {$[38]$} & {$[43]$} & {$[1]$} & {$[44]$} & {$[3]$} & {$[48]$} & {$[36]$} & {$[49]$} & {$[45]$} & {$[47]$} & {$[35]$} & {$[39]$} & {$[40]$} & {$[34]$} & {$[2]$} & {$[46]$} \\
\hline
\end{tabular}

8 Personalise programme to individual 5

$x \times x \times$

a. Multidimensional preassessment conducted by healthcare professional addressing health status, 27

cautions, contraindications and risks

\section{b. Pre-programme}

assessment by trainers to enable individualisation of programme

c. Adapt programme

content to personal situation $28 \quad x \quad x \quad x$
and goals

d. Evaluate programme

effects on individual, including satisfaction, functional gains, personal

goals, resource use, energy

levels

e. Supplement group classes with individual sessions

f. Sub-divide group classes according to disability levels

g. In group classes, conduct functional strengthening exercises together to allow individual monitoring

h. Intensity should be adjusted to the individual

i. Pre-programme ECG assessment for exercise level

j. If maximal heart-rate is unknown, use low intensity

but increase training

frequency/duration

k. Shorter, more frequent exercise for frail or deconditioned

I. Use of memory aids where necessary

9 Ensure dosage is sufficient to establish and maintain benefits

$31 \quad x$

a. At least 3 days/week physical exercise

b. 20-30 minutes daily moderate intensity physical 32 exercise

c. Progression: increase load / required effort over time

d. Aerobic exercise 20-60 minutes, 3-7 days/week; continuous or accumulated 
Table 3 Proximal objectives and strategies for programme delivery (Continued)

\begin{tabular}{|c|c|c|c|c|c|c|c|c|c|c|c|c|c|c|c|c|c|c|c|}
\hline & $\begin{array}{l}\text { f. Strengthening exercises: } \\
4-10 \text { types, 2-3 days/week }\end{array}$ & 3 & 3 & & & $x$ & & & & & & & & $x$ & & $x$ & & & \\
\hline & $\begin{array}{l}\text { g. Flexibility exercises: } 2-3 \\
\text { days/week }\end{array}$ & 3 & 2 & & & $x$ & & & & & & & & & & $x$ & & & \\
\hline & $\begin{array}{l}\text { h. Coordination \& balance } \\
\text { exercises: } 2-3 \text { days/week }\end{array}$ & 3 & 2 & & & $x$ & & & & & & & & & & $x$ & & & \\
\hline & $\begin{array}{l}\text { i. Upper limb exercises } 1 \\
\text { hour, } 6 \text { days/week }\end{array}$ & 3 & 1 & & $x$ & & & & & & & & & & & & & & \\
\hline & $\begin{array}{l}\text { j. Warm-up: } 15-20 \text { minutes } \\
\text { including range of } \\
\text { movement and large muscle } \\
\text { group activity }\end{array}$ & 2 & 2 & & & & & & & & & & & $x$ & $x$ & & & & \\
\hline & $\begin{array}{l}\text { k. Aerobic warm-up and } \\
\text { cool-down, 3-5 minutes at } \\
\text { lower intensity }\end{array}$ & 2 & 2 & & & $x$ & & & & & & & & $x$ & & & & & \\
\hline & $\begin{array}{l}\text { I. Aerobic: up to } 10 \text { exercises } \\
\text { alternating cardiovascular \& } \\
\text { local muscle endurance }\end{array}$ & 3 & 2 & & & & & & & & & & & $x$ & $x$ & & & & \\
\hline & $\begin{array}{l}\mathrm{m} \text {. Include home exercises } \\
\text { to increase dose }\end{array}$ & 3 & 4 & & & $x$ & $x$ & & & & $x$ & & & $x$ & & & & & \\
\hline 10 & $\begin{array}{l}\text { Structure programme to } \\
\text { facilitate ongoing regular } \\
\text { physical exercise }\end{array}$ & & 4 & $x$ & & $x$ & & & & & & & & $x$ & & & & $x$ & \\
\hline & $\begin{array}{l}\text { a. Pre-programme contact } \\
\text { to discuss any programme } \\
\text { barriers }\end{array}$ & 3 & 3 & & & $x$ & & & & & & & & $x$ & & $x$ & & & \\
\hline & $\begin{array}{l}\text { b. Peer/volunteer to } \\
\text { accompany to first one or } \\
\text { two sessions }\end{array}$ & 3 & 1 & & & & & & & & & & & $x$ & & & & & \\
\hline & $\begin{array}{l}\text { c. Minimal use of } \\
\text { equipment to facilitate } \\
\text { home practice }\end{array}$ & 3 & 1 & & & & & & & & & & & $x$ & & & & & \\
\hline & $\begin{array}{l}\text { d. Promote family / carer } \\
\text { involvement }\end{array}$ & 1 & 10 & $x$ & & $x$ & $x$ & & & & $x$ & $x$ & $x$ & & $x$ & $x$ & $x$ & $x$ & \\
\hline & e. Use peer mentoring & 2 & 2 & & & & & & & & $x$ & & & $x$ & & & & & \\
\hline & $\begin{array}{l}\text { f. Use group format for social } \\
\text { support }\end{array}$ & 3 & 1 & & & & & & & & & & & $x$ & & & & & \\
\hline & $\begin{array}{l}\text { g. Provide opportunities to } \\
\text { socialise before and after } \\
\text { training }\end{array}$ & 3 & 2 & & & & & & & & & & & $x$ & $x$ & & & & \\
\hline & $\begin{array}{l}\text { h. Use of mixed media } \\
\text { including internet-based and } \\
\text { tele-training }\end{array}$ & 2 & 4 & & & & & & & & $x$ & & & & $x$ & & & $x$ & $x$ \\
\hline & $\begin{array}{l}\text { i. Locate at home or centre } \\
\text { according to personal } \\
\text { circumstances / preferences }\end{array}$ & 1 & 5 & $x$ & & & $x$ & & $x$ & & & & & & $x$ & & & $x$ & \\
\hline & $\begin{array}{l}\text { j. Locate in own residential } \\
\text { environment }\end{array}$ & 1 & 3 & & & & & $x$ & & $x$ & & $x$ & & & & & & & \\
\hline & $\begin{array}{l}\text { k. Provision of transport } \\
\text { where necessary, or locate } \\
\text { near good public transport } \\
\text { links }\end{array}$ & 3 & 5 & $x$ & & $x$ & & & & & & & $x$ & $x$ & $x$ & & & & \\
\hline & I. Convenient time & 3 & 1 & & & $x$ & & & & & & & & & & & & & \\
\hline & $\begin{array}{l}\mathrm{m} \text {. Ongoing programme } \\
\text { provision }\end{array}$ & 3 & 2 & & & $x$ & & & & & & & & $x$ & & & & & \\
\hline & $\begin{array}{l}\text { n. Sign-post to other } \\
\text { relevant services / facilities }\end{array}$ & 3 & 4 & $x$ & & & & & & & $x$ & & $x$ & & & & & $x$ & \\
\hline
\end{tabular}


Table 3 Proximal objectives and strategies for programme delivery (Continued)

\begin{tabular}{|c|c|c|c|c|c|c|c|c|c|}
\hline 11 & $\begin{array}{l}\text { Ensure adequate staffing } \\
\text { numbers to provide safe } \\
\text { and effective training }\end{array}$ & & 2 & & $x$ & & $x$ & & \\
\hline & $\begin{array}{l}\text { a. Instructor: participant ratio: } \\
1: 3 \text { to } 1: 5\end{array}$ & 3 & 1 & $x$ & & & & & \\
\hline & $\begin{array}{l}\text { b. Instructor: participant ratio: } \\
\text { up to 1:8 depending on mix } \\
\& \text { time since started exercising }\end{array}$ & 3 & 2 & & & & $x$ & $x$ & \\
\hline & $\begin{array}{l}\text { c. Supernumerary volunteers } \\
\text { or trainees to take part in } \\
\text { sessions }\end{array}$ & 3 & 1 & & & & $x$ & & \\
\hline 12 & $\begin{array}{l}\text { Ensure staff are adequately } \\
\text { trained for client group }\end{array}$ & & 1 & & & & $x$ & & \\
\hline & $\begin{array}{l}\text { a. Delivered by instructors } \\
\text { with knowledge and training } \\
\text { in exercise and stroke }\end{array}$ & 3 & 3 & $x$ & & $x$ & $x$ & & \\
\hline & $\begin{array}{l}\text { b. Provide in-service training } \\
\text { to instructors }\end{array}$ & 3 & 1 & & & & $x$ & & \\
\hline & $\begin{array}{l}\text { c. Ensure stroke-awareness } \\
\text { training of frontline staff in } \\
\text { course venue }\end{array}$ & 3 & 1 & & & & $x$ & & \\
\hline 13 & $\begin{array}{l}\text { Integrate programme into } \\
\text { stroke pathway }\end{array}$ & & 3 & & $x$ & & $x$ & & $x$ \\
\hline & $\begin{array}{l}\text { a. Develop partnership } \\
\text { agreements between } \\
\text { stakeholders }\end{array}$ & 3 & 1 & & & & $x$ & & \\
\hline & $\begin{array}{l}\text { b. Referral by healthcare } \\
\text { practitioner using clear } \\
\text { eligibility criteria }\end{array}$ & 3 & 2 & $x$ & & & $x$ & & \\
\hline & $\begin{array}{l}\text { c. Encourage referring } \\
\text { practitioner to visit } \\
\text { programme }\end{array}$ & 3 & 1 & & & & $x$ & & \\
\hline & $\begin{array}{l}\text { d. Established procedures for } \\
\text { transferring responsibilities } \\
\text { from referrers to trainers }\end{array}$ & 3 & 2 & & & & $x$ & $x$ & \\
\hline & $\begin{array}{l}\text { e. Ongoing communication } \\
\text { with (and feedback to) other } \\
\text { stakeholders including } \\
\text { healthcare professionals, } \\
\text { service commissioners, local } \\
\text { stroke networks }\end{array}$ & 3 & 2 & & & & $x$ & $x$ & \\
\hline & $\begin{array}{l}\text { f. Referral for other } \\
\text { treatments where } \\
\text { appropriate }\end{array}$ & 3 & 2 & & & & $x$ & $x$ & \\
\hline 14 & $\begin{array}{l}\text { Ensure adequate } \\
\text { programme governance }\end{array}$ & & 1 & & & & $x$ & & \\
\hline & $\begin{array}{l}\text { a. Oversight by management } \\
\text { group }\end{array}$ & 3 & 1 & & & & $x$ & & \\
\hline & $\begin{array}{l}\text { b. Plan for programme } \\
\text { evaluation }\end{array}$ & 3 & 2 & $x$ & & & $x$ & & \\
\hline & $\begin{array}{l}\text { c. Use procedures for } \\
\text { recording and reporting } \\
\text { adverse events }\end{array}$ & 3 & 2 & $x$ & & & $x$ & & \\
\hline & $\begin{array}{l}\text { d. Follow data protection } \\
\text { procedures }\end{array}$ & 3 & 1 & & & & $x$ & & \\
\hline & $\begin{array}{l}\text { e. Obtain and check ongoing } \\
\text { consent }\end{array}$ & 3 & 2 & $x$ & & & $x$ & & \\
\hline
\end{tabular}

$\mathrm{Ev}=$ highest level of evidence presented for strategy.

$\mathrm{n}=$ number of guidelines explicitly including objective or strategy.

$\mathrm{Au}=$ Australia; $\mathrm{Ca}=\mathrm{Canada} ; \mathrm{Eu}=$ Europe; $\mathrm{Ne}=$ Netherlands, $\mathrm{NZ}=$ New Zealand; $\mathrm{SA}=$ South Africa; $\mathrm{Si}=$ Singapore . 
linkages were incorporated into the synthesis. Deliveryrelated objectives were formulated on the basis of themes identified during data extraction and analysis, and so delivery strategies were automatically linked to objectives. Some strategies were thought likely to serve more than one objective but, to avoid duplication, each strategy was assigned to a single objective in the synthesis.

\section{Discussion}

This synthesis, which we refer to as STRIDES (STroke Rehabilitation Intervention-Development Evidence Synthesis), presents an account of the many components that could be included in community-based exercise programmes for stroke survivors. STRIDES represents an exploratory attempt to develop a method of integrating diverse guidelines into a coherent and clinically applicable synthesis. It is novel in a number of respects: selectively abstracting from the guidelines only those recommendations relevant to a particular clinical application; including recommendations that relate not only to the content of the intervention but also to the way it is delivered and to other contextual factors that may significantly influence outcomes; and presenting recommendations in a format particularly suited to programme development. This combination of specificity, inclusivity and integrative restructuring adds value to the published guidelines by providing an account that is more tailored to particular needs and users, and provides a range of solutions to programme delivery issues, which may be selected according to local contexts and requirements. These characteristics can encourage guideline implementation by presenting relevant recommendations in a usable format, and addressing the range of organisational and service delivery issues that programme commissioners and practitioners must deal with.

The synthesis can be used for several purposes. First, to help plan an effective intervention by providing best evidenced suggestions addressing content, delivery issues and other contextual factors. We are currently assessing the value of this synthesis by applying it in the development of an intervention manual for a clinical trial. The synthesis will inform not only the content of the programme but also organisational planning, practitioner training and personalisation of the intervention. By providing guidance on all of these issues and relating them to particular objectives, the synthesis may be useful to others planning similar programmes and so could encourage better uptake of relevant best practice recommendations. Although the STRIDES approach requires further development, its essential components could be applied in the planning of other rehabilitation programmes and other forms of complex intervention. Its feasibility and effectiveness for the purpose requires further evaluation, including resource use compared to "de novo" development of an application-specific guideline.
Second, the synthesis can provide a checklist to evaluate the congruence of existing programmes with best-practice guidelines. We have employed this synthesis to analyse the content of a stroke exercise-based programme manual [50], and the content of exercise programmes informed by the manual (unpublished data). This has allowed us to establish the extent to which this programme meets best-practice guidelines, both in its intended form [50] and its real-world application. The synthesis also enabled identification of potentially novel aspects of the programme. This type of application could be elaborated by developing quality standards, based on the recommendations in the synthesis, to assist formative evaluation of best-practice adherence [51].

The synthesis also enables identification of limitations in guidelines that may affect their implementation. For example, much of cited evidence in this synthesis is derived from studies involving only ambulatory stroke survivors with mild to moderate disabilities, and there is very little reference to those with more severe physical, cognitive and language problems, for whom different strategies may be more feasible or effective. Guideline recommendations that do not take explicit account of the severity of patients' or clients' impairments may be rejected by practitioners as impossible to implement [52]. Thus, further research is required to identify strategies that are appropriate for those with more severe impairments. More stroke-specific research is also needed to support recommended strategies concerned with exercise psychology and behaviour change. This is particularly pertinent to those focusing on motivation and self-management skills, both of which may be key to the target outcome of increasing life-long activity levels $[53,54]$. The inconsistencies between guidelines that were found in some areas may reflect differences in expert opinion where the evidence is scant or of low quality. Robust evidence in these areas may be difficult to obtain [35] and is likely to be context-dependent, but the synthesis includes alternative options suggested in guidelines, and users may choose those they consider most appropriate to their context.

The Intervention Mapping concept provided a useful framework for the synthesis because of its focus on generating an organised programme plan comprising descriptions of target outcomes, proximal objectives and strategies. Since the guidelines differed in scope and language, the imposition of a common terminology was essential. It was necessary to infer proximal objectives for some strategies where these were not explicitly stated. Some degree of conceptual innovation is inevitable in qualitative synthesis [55], and we sought to validate this process by involving several reviewers in the development of themes and terminology, and in the classification of strategies under particular objectives. Other conceptual structures could be used to inform the synthesis, for example the World Health Organisation's 
International Classification of Functioning and Disability (ICF) [56]. This employs a biopsychosocial perspective to generate a structured and comprehensive description of the many components of health-state, classified under the domains of body function and structure, activities and participation, and personal and environmental factors. Thus, it can be used to help describe and develop complex health interventions [57]. The ICF emphasises the importance of context and identifies many that relate to service provision, social support and other environmental factors. One of the guidelines included in STRIDES employed the ICF to structure its recommendations [3], and it informed our own thinking during the synthesis process. Sub-sets of ICF classifications have been developed specifically for stroke $[58,59]$ and other health conditions (e.g., [60,61]), and some studies have suggested personal contextual factors that are not currently classified by the ICF $[59,62,63]$. This synthesis could be further developed by using the ICF to assess the scope of included guidelines, and to identify areas where new recommendations could be developed.

The Intervention Mapping approach recommends that intervention descriptions specify theoretical mechanisms by which strategies achieve objectives and target outcomes [27]. These were provided in some of the guidelines. Our synthesis could be enhanced by including theoretical mechanisms, as these could assist strategy selection when programmes are being developed with more limited target outcomes, for instance focusing on post-stroke mobility or falls prevention. Information about proposed mechanisms of action might be particularly important in such circumstances, where there may be synergistic relationships between objectives, and strategies may serve multiple objectives.

A number of other issues arose during the synthesis process with implications for its future development and use. Several of the included guidelines were found through free text internet searches, rather than bibliographic or guideline databases. This underlines the value of a broad search strategy, including the use of alternative search terms and databases [64]. Our search may have missed some relevant publications that, along with more recent editions of existing guidelines, could alter the synthesis recommendations. Two guidelines were not accessed because they were not freely available, but we reviewed summaries of these on www.guidelines.gov and found no recommendations additional to those in the synthesis. At least one of the included guidelines has recently been updated [4]; however, inspection of the updated version revealed further support for strategies included in the synthesis, rather than suggesting additional ones.

We did not formally rate the quality of included guidelines, though this was variable. Several provided limited or no descriptions of how the evidence they cited had been selected $[35,40,42]$, and so could have been subject to bias. Also, some recommendations were supported by trials of interventions that involved multiple strategies where the effectiveness of a particular component, such as the use of cool-down activities, is unknown [35]. Particularly where there are discrepancies or doubts about particular recommendations, some assessment of the methodological quality of guidelines may help users prioritise recommendations for implementation. Instrument such as the Appraisal of Guidelines, REsearch and Evaluation (AGREE) framework are available to facilitate quality assessment [65]. A review of stroke practice guidelines [66] using the AGREE framework with versions of six of the guidelines included in our synthesis concluded that four of them $[2,45,47,48]$ were of good quality. However, high quality evidence does not necessarily imply relevance or applicability to a particular application or population, and guidelines using lower methodological quality evidence may contain valuable relevant recommendations. Consequently, we would argue that quality appraisal should not be the only criterion used to judge eligibility for inclusion in the synthesis.

\section{Conclusions}

The implementation of practice guidelines may be enhanced by generating accounts that are specific to a particular application and which address not only the content of interventions but also the way they should be delivered, along with other contextual factors that may influence their effectiveness. STRIDES does this by selecting and synthesising relevant best practice recommendations from a range of guidelines, and providing a structured account that can assist those responsible for the development of new programmes and evaluation of existing ones. By including both well-evidenced recommendations and those based on expert consensus, this approach creates a comprehensive description that covers all aspects of programme design. The synthesis also highlights where there is legitimate scope for variation in practice. The methods we have employed require further development, but could be applied to other complex interventions, not only for stroke but for a variety of health conditions.

\section{Additional files}

\section{Additional file 1: Guideline search strategies. \\ Additional file 2: Programme proximal objectives and strategies, with guideline page/section references.}

Additional file 3: Proximal objectives, strategies and evidence levels cited in guidelines.

\section{Competing interests}

CK is a member of the RCP Intercollegiate Stroke Working Party responsible for development of their 2012 stroke guidelines.

An abstract of the early stages of this study has been published [67]. 


\section{Authors' contributions}

LP was lead author and reviewer; CA advised on Intervention Mapping and the conceptualisation of the review, and contributed to drafts of paper; AF contributed to drafts of the paper; VG and CK were involved in the review synthesis and contributed to drafts of the paper; RT helped conceptualise the review and contributed to drafts of the paper; SD was involved in conceptualising and conducting the synthesis, and contributed to drafts of the paper. All authors read and approved the final manuscript.

\section{Acknowledgements}

Thanks to the reviewers of the manuscript for valuable comments and suggestions. This paper presents independent research funded by the National Institute for Health Research (NIHR) Collaboration for Leadership in Applied Health Research and Care (CLAHRC) for the South West Peninsula. The views expressed in this publication are those of the authors and not necessarily those of the NHS, the NIHR or the Department of Health in England.

\section{Author details}

${ }^{1}$ University of Exeter Medical School, Veysey Building, Salmon Pool Lane, Exeter EX2 4SG, UK. ²University of Exeter Medical School, St Luke's Campus, Magdalen Rd, Exeter, UK. ${ }^{3}$ Academic Unit of Elderly Care and Rehabilitation (University of Leeds), Bradford Institute for Health Research, Bradford Royal Infirmary, Duckworth Lane, Bradford, UK. ${ }^{4}$ Centre for Research in Rehabilitation, Brunel University London, Mary Seacole Building, Kingston Lane, Middlesex, UB8 3PH, UK.

Received: 3 June 2013 Accepted: 28 September 2013

Published: 1 October 2013

\section{References}

1. Evidence-Based Review of Stroke Rehabilitation. www.ebrsr.com/.

2. Department of Veteran Affairs: VA/DOD Clinical practice guideline for the management of stroke rehabilitation. J Rehabil Res Dev 2010, 47(9):1-43.

3. van Peppen RPS, Kwakkel G, Harmeling-van der Wel BC, Kollen BJ, Hobbelen JSM, Buurke JH: KNGF Clinical Practice Guideline for physical therapy in patients with stroke. Review of the evidence. [Translation 2008]. Nederlands Tijdschrift voor Fysiotherapie 2004, 114(5):1-248.

4. Intercollegiate Stroke Working Party: National Clinical Guideline for Stroke. 4th edition. London: Royal College of Physicians; 2012.

5. Michie S, Lester K: Words matter: increasing the implementation of clinical guidelines. Qual Saf Health Care 2005, 14(5):367-370.

6. Donnellan C, Sweetman S, Shelley E: Implementing clinical guidelines in stroke: a qualitative study of perceived facilitators and barriers. Health Policy 2013, 111:234-244.

7. Rycroft-Malone J, Harvey G, Seers K, Kitson A, McCormack B, Titchen A: An exploration of the factors that influence the implementation of evidence into practice. J Clin Nurs 2004, 13(8):913-924.

8. Francke AL, Smit MC, de Veer AJ, Mistiaen P: Factors influencing the implementation of clinical guidelines for health care professionals: a systematic meta-review. BMC Med Inform Decis Mak 2008, 8:38.

9. Shekelle P, Woolf S, Grimshaw JM, Schunemann HJ, Eccles MP: Developing clinical practice guidelines: reviewing, reporting, and publishing guidelines; updating guidelines; and the emerging issues of enhancing guideline implementability and accounting for comorbid conditions in guideline development. Implement Sci 2012, 7:62.

10. Gagliardi AR, Brouwers MC, Palda VA, Lemieux-Charles L, Grimshaw JM: How can we improve guideline use? A conceptual framework of implementability. Implement Sci 2011, 6:26.

11. Fervers B, Burgers JS, Haugh MC, Latreille J, Mlika-Cabanne N, Paquet L, Coulombe M, Poirier M, Burnand B: Adaptation of clinical guidelines: literature review and proposition for a framework and procedure. Int I Qual Health C 2006, 18(3):167-176

12. ADAPTE Collaboration: The ADAPTE Process: Resource Toolkit for Guideline adaptation. Version 2.0 edition; 2009. http://www.g-i-n.net.

13. Dagenais S, Tricco AC, Haldeman S: Synthesis of recommendations for the assessment and management of low back pain from recent clinical practice guidelines. Spine J 2010, 10(6):514-529.

14. Wottrich AW, von Koch $L$, Tham $K$ : The meaning of rehabilitation in the home environment after acute stroke from the perspective of a multiprofessional team. Phys Ther 2007, 87(6):778-788.
15. Jones F, Livingstone E, Hawkes L: 'Getting the balance between encouragement and taking over' - reflections on using a new stroke self-management programme. Physiother Res Int 2013, 18(2):91-99. doi: 10.1002/pri.1531. Epub 2012 Jul 1005.

16. Hillier S, Inglis-Jassiem G: Rehabilitation for community-dwelling people with stroke: home or centre based? A systematic review. Int J Stroke 2010, 5(3):178-186.

17. Wang PP, Badley EM, Gignac M: Exploring the role of contextual factors in disability models. Disabil Rehabil 2006, 28(2):135-140.

18. Resnik $L$, Jensen GM: Using clinical outcomes to explore the theory of expert practice in physical therapy. Phys Ther 2003, 83(12):1090-1106.

19. Campbell NC, Murray E, Darbyshire J, Emery J, Farmer A, Griffiths F, Guthrie $\mathrm{B}$, Lester $\mathrm{H}$, Wilson $\mathrm{P}$, Kinmonth AL: Designing and evaluating complex interventions to improve health care. BMJ 2007, 334(7591):455-459.

20. Medical Research Council: Developing and evaluating complex interventions: new guidance. London: Medical Research Council; 2008.

21. Rycroft-Malone J, McCormack B, Hutchinson AM, DeCorby K, Bucknall TK, Kent B, Schultz A, Snelgrove-Clarke E, Stetler CB, Titler M, et al: Realist synthesis: illustrating the method for implementation research. Implement Sci 2012, 7:33.

22. Pawson R, Tilley N: Realistic evaluation. London: Sage; 1997:235.

23. Hickey A, Horgan F, O'Neill D, McGee H, Inasc OB: Community-based poststroke service provision and challenges: a national survey of managers and inter-disciplinary healthcare staff in Ireland. BMC Health Serv Res 2012, 12(1):111.

24. Life after stroke: Participating in community exercise and fitness. http://www. improvement.nhs.uk/stroke/CommunityStrokeResource/CSRLifeafterstroke/ CSRLifeafterstrokeexercisepoststroke/tabid/226/Default.aspx.

25. Best C, van Wijck F, Dennis J, Smith M, Donaghy M, Fraser H, DinanYoung S, Mead G: A survey of community exercise programmes for stroke survivors in Scotland. Health Soc Care Community 2012, 20(4):400-411.

26. Eng J, Chu KKM, Dawson A, Carswell A, Hepburn K: A community-based group exercise program for persons with chronic stroke. Med Sci Sports Exerc 2003, 35(8):1271-1278.

27. Bartholomew LK, Parcel GS, Kok G: Intervention mapping: a process for developing theory- and evidence-based health education programs. Health Educ Behav 1998, 25(5):545-563.

28. Michie S: Designing and implementing behaviour change interventions to improve population health. J Health Serv Res Policy 2008, 13(Suppl 3):64-69.

29. Schmid AA, Andersen J, Kent T, Williams LS, Damush TM: Using intervention mapping to develop and adapt a secondary stroke prevention program in Veterans Health Administration medical centers. Implement Sci 2010, 5:97.

30. Lloyd JJ, Logan S, Greaves CJ, Wyatt KM: Evidence, theory and contextusing intervention mapping to develop a school-based intervention to prevent obesity in children. Int J Behav Nutr Phys Act 2011, 8:73.

31. McEachan RR, Lawton RJ, Jackson C, Conner M, Lunt J: Evidence, theory and context: using intervention mapping to develop a worksite physical activity intervention. BMC Public Health 2008, 8:326.

32. Caspersen CJ, Powell KE, Christenson GM: Physical activity, exercise, and physical fitness: definitions and distinctions for health-related research. Public Health Rep 1985, 100(2):126-131.

33. King N: Using templates in the thematic analysis of text. In Essential Guide to Qualitative Methods in Organizational Research. Edited by Cassell C, Symon G. London: Sage; 2004:256-270.

34. Miller EL, Murray L, Richards L, Zorowitz RD, Bakas T, Clark P, Billinger SA: Comprehensive overview of nursing and interdisciplinary rehabilitation care of the stroke patient: a scientific statement from the American Heart Association. Stroke 2010, 41(10):2402-2448.

35. Best C, van Wijck F, Dinan-Young S, Dennis J, Smith M, Frazer H, Donaghy M, Meda G: Best Practice Guidance for the Development of Exercise after Stroke Services in Community Settings. University of Edinburgh; 2010.

36. Bryer A, Connor M, Haug P, Cheyip B, Staub H, Tipping B, Duim W Pinkney-Atkinson $V$ : South African guideline for management of ischaemic stroke and transient ischaemic attack 2010: a guideline from the South African Stroke Society (SASS) and the SASS Writing Committee. S Afr Med J 2010, 100(11 Pt 2):747-778.

37. Canadian Stroke Network: Canadian Best Practice Recommendations for Stroke Care. Ottawa: Canadian Stroke Network; 2010.

38. Consensus Panel on the Management of the Hemiplegic Arm and Hand: Management of the Post Stroke Arm and Hand: Treatment Recommendations of the 2001 Consensus Panel. Hamilton, Ontario: Heart and Stroke Foundation of Ontario; 2001. 
39. Duncan PW, Zorowitz R, Bates B, Choi JY, Glasberg JJ, Graham GD, Katz RC, Lamberty K, Reker D: Management of adult stroke rehabilitation care: a clinical practice guideline. Stroke 2005, 36(9):e100-e143.

40. Gordon NF, Gulanick M, Costa F, Fletcher G, Franklin BA, Roth EJ, Shephard T: Physical activity and exercise recommendations for stroke survivors: an American Heart Association scientific statement. Stroke 2004, 35(5):1230-1240

41. National Stroke Foundation: Clinical Guidelines for Stroke 2010. Melbourne: National Stroke Foundation; 2010.

42. Ontario Stroke Network: Guidelines for Community Based Exercise Programs for People with Stroke. Toronto: Ontario Stroke Network; 2010.

43. Ottawa Panel: Ottawa panel evidence-based clinical practice guidelines for post-stroke rehabilitation. Top Stroke Rehabil 2006, 13(2):1-269.

44. Quinn TJ, Paolucci S, Sunnerhagen KS, Sivenius J, Walker MF, Toni D, Lees KR: Evidence-based stroke rehabilitation: an expanded guidance document from the european stroke organisation (ESO) guidelines for management of ischaemic stroke and transient ischaemic attack 2008. J Rehabil Med 2009, 41(2):99-111.

45. Intercollegiate Stroke Working Party: National Clinical Guideline for Stroke. 3rd edition. London: Royal College of Physicians; 2008.

46. Schwamm LH, Audebert HJ, Amarenco P, Chumbler NR, Frankel MR, George MG, Gorelick PB, Horton KB, Kaste M, Lackland DT, et al: Recommendations for the implementation of telemedicine within stroke systems of care: a policy statement from the American Heart Association. Stroke 2009, 40(7):2635-2660.

47. SIGN: Management of patients with stroke or TIA: assessment, investigation, immediate management and secondary prevention. A national clinical guideline. Edinburgh: Scottish Intercollegiate Guidelines Network; 2008.

48. Stroke Foundation of New Zealand and New Zealand Guidelines Group: Clinical Guidelines for Stroke Management 2010. Wellington: Stroke Foundation of New Zealand; 2010.

49. Venketasubramanian N, Pwee KH, Chen CP: Singapore ministry of health clinical practice guidelines on stroke and transient ischemic attacks. Int J Stroke 2011, 6(3):251-258.

50. Balchin T: The Successful Stroke Survivor: A new guide to functional recovery from stroke. Lingfield, UK: ARNI Trust; 2011.

51. Stetler CB, Legro MW, Wallace CM, Bowman C, Guihan M, Hagedorn H, Kimmel B, Sharp ND, Smith JL: The role of formative evaluation in implementation research and the QUERI experience. J Gen Intern Med 2006, 21(Suppl 2):S1-S8.

52. Bayley MT, Hurdowar A, Richards CL, Korner-Bitensky N, Wood-Dauphinee S, Eng JJ, McKay-Lyons M, Harrison E, Teasell R, Harrison M, et al: Barriers to implementation of stroke rehabilitation evidence: findings from a multisite pilot project. Disabil Rehabil 2012, 34(19):1633-1638.

53. Shaughnessy M, Resnick BM, Macko RF: Testing a model of post-stroke exercise behavior. Rehabil Nurs 2006, 31(1):15-21.

54. Michie S, Abraham C, Whittington C, McAteer J, Gupta S: Effective techniques in healthy eating and physical activity interventions: a metaregression. Health Psychol 2009, 28(6):690-701.

55. Thomas J, Harden A: Methods for the thematic synthesis of qualitative research in systematic reviews. BMC Med Res Methodol 2008, 8:45.

56. WHO: International Classification of Functioning, Disability and Health. Geneva: World Health Organisation; 2001.

57. Taylor WJ, Geyh S: A rehabilitation framework: the international classification of functioning, disability and health. In Interprofessional Rehabilitation: A Person-Centred Approach. Edited by Dean S, Siegert R, Taylor WJ. Chichester: John Wiley; 2012.

58. Geyh S, Cieza A, Schouten J, Dickson H, Frommelt P, Omar Z, Kostanjsek N, Ring H, Stucki G: ICF core sets for stroke. J Rehabil Med 2004, 44(Suppl):135-141.

59. Glassel A, Coenen M, Kollerits B, Cieza A: Validation of the extended ICF core set for stroke from the patient perspective using focus groups. Disabil Rehabil 2012, 34(2):157-166.

60. Scheuringer M, Stucki G, Huber EO, Brach M, Schwarzkopf SR, Kostanjsek N, Stoll T: ICF Core Set for patients with musculoskeletal conditions in early post-acute rehabilitation facilities. Disabil Rehabil 2005, 27(7-8):405-410.

61. Wildner M, Quittan M, Portenier L, Wilke S, Boldt C, Stucki G, Kostanjsek N, Grill E: ICF Core Set for patients with cardiopulmonary conditions in early post-acute rehabilitation facilities. Disabil Rehabil 2005, 27(7-8):397-404.

62. Glassel A, Kirchberger I, Kollerits B, Amann E, Cieza A: Content validity of the extended ICF core set for stroke: an international delphi survey of physical therapists. Phys Ther 2011, 91(8):1211-1222.
63. Glassel A, Kirchberger I, Linseisen E, Stamm T, Cieza A, Stucki G: Content validation of the international classification of functioning, disability and health (ICF) core set for stroke: the perspective of occupational therapists. Can J Occup Ther 2010, 77(5):289-302.

64. Haase A, Follmann M, Skipka G, Kirchner H: Developing search strategies for clinical practice guidelines in SUM search and google scholar and assessing their retrieval performance. BMC Med Res Methodol 2007, 7:28.

65. The AGREE Collaboration: Development and validation of an international appraisal instrument for assessing the quality of clinical practice guidelines: the AGREE project. Qual Saf Health Care 2003, 12(1):18-23.

66. Hurdowar A, Graham ID, Bayley M, Harrison M, Wood-Dauphinee S, Bhogal S: Quality of stroke rehabilitation clinical practice guidelines. J Eval Clin Pract 2007, 13(4):657-664.

67. Goodwin V, Poltawski L, Kilbride C, Abraham C, Taylor R, Forster A, Dean S: Exercise for stroke survivors: a synthesis of evidence-based guidelines. J Aging Phys Act 2012, 20:245.

\section{doi:10.1186/1748-5908-8-115}

Cite this article as: Poltawski et al: Synthesising practice guidelines for the development of community-based exercise programmes after stroke. Implementation Science 2013 8:115.

\section{Submit your next manuscript to BioMed Central and take full advantage of:}

- Convenient online submission

- Thorough peer review

- No space constraints or color figure charges

- Immediate publication on acceptance

- Inclusion in PubMed, CAS, Scopus and Google Scholar

- Research which is freely available for redistribution

Submit your manuscript at www.biomedcentral.com/submit
C Biomed Central 